\title{
Surface subgroups from homology
}

\author{
DANNY CALEGARI
}

Let $G$ be a word-hyperbolic group, obtained as a graph of free groups amalgamated along cyclic subgroups. If $H_{2}(G ; \mathbb{Q})$ is nonzero, then $G$ contains a closed hyperbolic surface subgroup. Moreover, the unit ball of the Gromov-Thurston norm on $\mathrm{H}_{2}(G ; \mathbb{R})$ is a finite-sided rational polyhedron.

20F65, 20F67; 57M07

\section{Introduction}

A famous question of Gromov (see Bestvina [2]) asks whether every one-ended nonelementary word-hyperbolic group contains a closed hyperbolic surface subgroup. Almost nothing is known about this question in general. Gordon-Long-Reid [7] answer the question affirmatively for Coxeter groups and some Artin groups.

Bestvina remarks that Gromov's question is inspired by the well known virtual Haken conjecture in 3-manifold topology. The case of 3-manifold groups is instructive. If $M$ is an aspherical 3 -manifold, every integral homology class in $H_{2}(M ; \mathbb{Z})$ is represented by an embedded surface $S$. If $S$ is not $\pi_{1}$-injective, Dehn's lemma (see Hempel [11, Chapter 4]) implies that $S$ can be compressed, reducing $-\chi(S)$. By the hypothesis that $M$ is aspherical, after finitely many compressions, one obtains a $\pi_{1}$-injective surface representing the given homology class.

For more general classes of groups, no tool remotely resembling Dehn's lemma exists. Nevertheless one can consider the following strategy. Let $X$ be a $K(G, 1)$, and let $A$ be a rational homology class in $H_{2}(X ; \mathbb{Q})$. Suppose one can find a map of a closed surface $f: S \rightarrow X$ with no spherical components, representing $n(S) A$ in $H_{2}(X)$ for some integer $n(S)$, that realizes the infimum of $-\chi(S) / n(S)$ over all surfaces and all integers $n$. Then $f_{*}: \pi_{1}(S) \rightarrow \pi_{1}(X)=G$ is injective. For, otherwise, one could find an essential loop $\alpha \subset S$ in the kernel of $f_{*}$ and (by Scott [13]) find a suitable finite cover $S^{\prime}$ of $S$ to which $\alpha$ lifts as an embedded loop. Then $f^{\prime}: S^{\prime} \rightarrow X$ could be compressed along $\alpha$, producing a new surface $S^{\prime \prime}$ representing $n\left(S^{\prime \prime}\right) A$ in homology, and satisfying $-\chi\left(S^{\prime \prime}\right) / n\left(S^{\prime \prime}\right)<-\chi(S) / n(S)$, contrary to hypothesis. The infimum of ratios $-\chi(S) / n(S)$ over all possible surfaces $S$ is called the Gromov-Thurston 
norm of the homology class $A$ (see Gromov [8] or Thurston [15] for an introduction to Gromov-Thurston norms and bounded cohomology). In words, if a map from a surface to $X$ realizes the Gromov-Thurston norm in a given projective homology class, it is injective.

It is therefore an intriguing question to understand for which groups $G$ and which homology classes in $H_{2}(G ; \mathbb{Q})$ one can find maps of surfaces (projectively) realizing the Gromov-Thurston norm. In this paper we show that if $G$ is a group obtained as a graph of free groups amalgamated along cyclic subgroups, and $A \in H_{2}(G ; \mathbb{Q})$ is a homology class with nonzero Gromov-Thurston norm, then some map of a surface to a $K(G, 1)$ realizes the Gromov-Thurston norm in the projective class of $A$, and therefore $G$ contains a closed hyperbolic surface subgroup. The method of proof is to localize the problem to finding norm minimizers for suitable relative homology classes in the free factors. The relative Gromov-Thurston norm (after normalization) turns out to be equal to the so-called stable commutator norm, which we introduced in [3] and studied in free groups in [4]. A consequence of the main theorem of [4] is that extremal surfaces for the stable commutator norm exist in every rational relative homology class in a free group. These extremal surfaces can be glued together to produce extremal (closed) surface subgroups in $G$. A more careful analysis reveals that the Gromov-Thurston norm on $G$ is piecewise rational linear, and if $G$ is word-hyperbolic, the unit ball is a finite-sided rational polyhedron.

\section{The scl norm}

\subsection{Commutator length}

If $G$ is a group and $g \in[G, G]$, the commutator length of $g$ (denoted $\operatorname{cl}(g)$ ) is the smallest number of commutators in $G$ whose product is equal to $G$, and the stable commutator length of $g$ (denoted $\operatorname{scl}(g)$ ) is the limit

$$
\operatorname{scl}(g)=\lim _{n \rightarrow \infty} \frac{\operatorname{cl}\left(g^{n}\right)}{n}
$$

Geometrically, $\operatorname{cl}(g)$ is the least genus of a surface group that bounds $g$ homologically. Since genus is not multiplicative under covers but Euler characteristic is, one can derive a formula for $\mathrm{scl}$ in terms of Euler characteristic; we give such a formula in Definition 2.2 below.

Stable commutator length is, in a sense to be made precise shortly, a kind of relative Gromov-Thurston norm.

The following material is largely drawn from [4, Section 2.4]. Also see the papers by the author [3, Section 2.6] and Bavard [1, Section 3]. 
Definition 2.1 Let $S$ be a compact orientable surface. Define

$$
\chi^{-}(S)=\sum_{S_{i}} \min \left(0, \chi\left(S_{i}\right)\right)
$$

where the sum is taken over connected components $S_{i}$ of $S$.

Definition 2.2 Let $G$ be a group. Let $g_{1}, \cdots, g_{m}$ be elements in $G$ (not necessarily distinct). Let $X$ be a connected CW complex with $\pi_{1}(X)=G$. Further, for each $i$, let $\gamma_{i}: S^{1} \rightarrow X$ be a loop in $X$ in the free homotopy class corresponding to the conjugacy class of $g_{i}$.

If $S$ is an orientable surface, a map $f: S \rightarrow X$ is admissible of degree $n(S)$ for some positive integer $n(S)$ if there is a commutative diagram

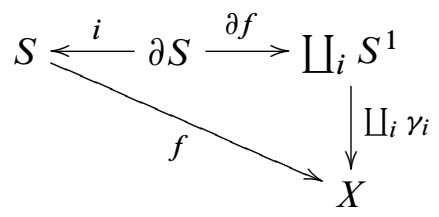

so that the homology class of $\partial f_{*}[\partial S]$ is equal to $n(S)$ times the fundamental class of $\bigsqcup_{i} S^{1}$ in $H_{1}$.

Then define

$$
\operatorname{scl}\left(g_{1}+g_{2}+\cdots+g_{m}\right)=\inf _{S} \frac{-\chi^{-}(S)}{2 n(S)}
$$

where the infimum is taken over all admissible maps of surfaces. If no admissible surfaces exist, $\operatorname{set} \operatorname{scl}\left(\sum_{i} g_{i}\right)=\infty$.

Remark 2.3 If $X$ has enough room (eg if $X$ is a manifold of dimension $>2$ ) then the maps $\gamma_{i}$ can be taken to be embeddings, and one can speak of the maps $\gamma_{i}$ and their images interchangeably. In this context, one can think of an admissible map as a map of pairs $(S, \partial S) \rightarrow\left(X, \cup_{i} \gamma_{i}\right)$ that wraps $\partial S$ around each $\gamma_{i}$ with total degree $n(S)$.

Remark 2.4 When $g \in[G, G]$, the value of $\operatorname{scl}(g)$ is the same with either definition above.

The function scl can be extended to integral group 1-chains, by the formula

$$
\operatorname{scl}\left(\sum n_{i} g_{i}\right):=\operatorname{scl}\left(\sum g_{i}^{n_{i}}\right)
$$


and extended to rational chains by linearity, and to real chains by continuity. It is finite exactly on group 1-chains that are boundaries of group 2-chains; in other words, scl defines a pseudo-norm on the real vector space $B_{1}(G ; \mathbb{R})$, hereafter denoted $B_{1}(G)$.

Notice that scl is, by construction, a homogeneous class function in each variable separately. If $H$ denotes the subspace of $B_{1}(G ; \mathbb{R})$ spanned by elements of the form $g-h g h^{-1}$ and $g^{n}-n g$ for $g, h \in G$ and $n \in \mathbb{Z}$, then scl descends to a pseudo-norm on $B_{1}(G) / H$.

\subsection{Comparison with Gromov and filling norms}

Let $C_{*}(G ; \mathbb{R})$ be the bar chain complex of a group (see eg Mac Lane [12, Chapter IV, Section 5] for details). In the sequel, the coefficient group $\mathbb{R}$ is understood where omitted. There is a natural basis for $C_{i}(G)$ in each dimension, and each $C_{i}(G)$ becomes a Banach space with respect to the natural $L^{1}$ norm. This norm induces a pseudo-norm on (group) homology, called the Gromov norm (or $L^{1}$ norm) defined by

$$
\|[A]\|_{1}=\inf _{C \in[A]}\|C\|_{1}
$$

where the infimum ranges over all cycles $C$ representing a homology class $[A]$.

If $X$ is a $K(G, 1)$, the norm on $H_{2}(G ; \mathbb{Q})$ may be calculated geometrically by the formula

$$
\|[A]\|_{1}=\inf _{S} \frac{-2 \chi^{-}(S)}{n(S)}
$$

where the infimum is taken over all closed oriented surfaces $S$ mapping to $X$ by $f: S \rightarrow X$ such that $f_{*}[S]=n(S)[A]$ for some integer $n(S)$, and then extended to $\mathrm{H}_{2}(G ; \mathbb{R})$ by continuity; see Gabai [5, Corollary 6.18]. Also compare with Definition 2.2 .

There is a natural norm on $B_{1}(G)$, called the (Gersten) filling norm, introduced in [6], defined by the formula

$$
\|A\|_{\partial}=\inf _{\partial C=A}\|C\|_{1}
$$

where $\|\cdot\|_{1}$ denotes the $L^{1}$ norm on group 2-chains. Let fill( $\left.\cdot\right)$ be the homogenization of $\|\cdot\|_{\partial}$; ie

$$
\operatorname{fill}\left(\sum t_{i} g_{i}\right)=\lim _{n \rightarrow \infty} \frac{\left\|\sum t_{i} g_{i}^{n}\right\|_{\partial}}{n}
$$

where $t_{i} \in \mathbb{R}$ and $g_{i} \in G$. Then fill descends to a function on $B_{1}(G) / H$ and satisfies

$$
\operatorname{scl}(A)=\frac{\operatorname{fill}(A)}{4}
$$


For $A=g$ for $g \in[G, G]$, this is proved in Bavard [1]; the general case follows basically the same argument and is found in [3, Section 2.6]. The factor of $\frac{1}{4}$ arises because fill counts triangles, whereas scl counts genus. This explains the sense in which scl can be thought of as a relative Gromov-Thurston norm.

\subsection{Extremal surfaces}

Given an integral chain $\sum n_{i} g_{i}$, an admissible surface is extremal if it realizes

$$
\operatorname{scl}\left(\sum n_{i} g_{i}\right)=\frac{-\chi^{-}(S)}{2 n(S)}
$$

The Rationality Theorem from [4], is the following:

Theorem 2.5 Rationality Theorem [4, page 15] Let $F$ be a free group.

(1) $\operatorname{scl}(g) \in \mathbb{Q}$ for all $g \in[F, F]$.

(2) Every integral chain $\sum n_{i} g_{i}$ in $B_{1}(F)$ bounds an extremal surface

(3) The function $\mathrm{scl}$ is piecewise rational linear on $B_{1}(F)$

(4) There is an algorithm to calculate scl on any finite dimensional rational subspace of $B_{1}(F)$

In fact, [4], bullet (2) merely says that every $g \in[F, F]$ rationally bounds an extremal surface, but the argument of the proof establishes the more general statement. The method of proof makes this clear: let $X$ be a handlebody with $\pi_{1}(X)=F$, and let $\gamma_{i}$ be loops in $X$ representing the free homotopy classes of the $g_{i}$. In [4] it is shown that there is a simple branched surface $\mathcal{B}$, with boundary mapping to $\cup_{i} \gamma_{i}$, that carries every admissible surface (after compression and homotopy). The function $-\chi^{-}$is a rational linear function of weights on $\mathcal{B}$, and therefore $-\chi^{-}$may be calculated on any rational class by solving a linear programming problem. An extremal vector obtained eg by the simplex method will be rational, and after scaling, is represented by an extremal surface.

The term "piecewise rational linear" deserves a brief explanation. A rational structure on a real vector space $V$ is a vector space $W$ over $\mathbb{Q}$ together with an isomorphism $V=W \otimes_{\mathbb{Q}} \mathbb{R}$. A convex function $f$ on a finite-dimensional vector space $V$ with a rational structure is said to be piecewise rational linear if it has the form $f(v)=$ $\sup _{i} f_{i}(v)$ for some finite collection of linear functions $f_{i}$ that are rational on $W$. A convex function on a (possibly infinite dimensional) vector space $V$ with a rational structure is piecewise rational linear if its restriction to each finite dimensional rational subspace is piecewise rational linear.

We will also use the following technical Lemma, which is Lemma 4.2 from [4]: 
Lemma 2.6 Let $S$ be a connected surface, and $f: S \rightarrow H$ an extremal surface rationally bounding $\gamma$. Then there is another extremal surface $f^{\prime}: S^{\prime} \rightarrow H$ rationally bounding $\gamma$, such that every component of $\partial S^{\prime}$ maps to $\gamma$ with positive degree.

The same argument shows that if $S$ bounds some collection $\sum \gamma_{i}$, one may replace $S$ if necessary by another extremal surface such that every map of a boundary component of $S$ to every component $\gamma_{i}$ has positive degree. Such an extremal surface is said to be positive. Hence in the sequel we will assume that all our extremal surfaces are positive.

From our perspective, the importance of extremal surfaces is the following:

Lemma 2.7 Let $f:(S, \partial S) \rightarrow\left(X, \cup_{i} \gamma_{i}\right)$ be an extremal surface for $\sum n_{i} g_{i}$. Then $f, S$ is incompressible and boundary incompressible. That is, $f_{*}: \pi_{1}(S) \rightarrow \pi_{1}(X)$ is injective, and if $\alpha \subset S$ is an essential immersed proper arc with endpoints on components $\partial_{i}, \partial_{j}$ of $\partial S$ both mapping to $\gamma_{k}$, there is no arc $\beta \subset \gamma_{k}$ so that $f(\alpha) \cup \beta$ is homotopically trivial in $X$.

Proof Suppose $\alpha \subset S$ represents a conjugacy class in the kernel of $f_{*}$. Since surface groups are LERF [13], there is a finite cover $S^{\prime}$ of $S$ to which $\alpha$ lifts as an embedded loop. The lifted map $f^{\prime}: S^{\prime} \rightarrow X$ is admissible, with $-\chi^{-}\left(S^{\prime}\right) / 2 n\left(S^{\prime}\right)=$ $-\chi^{-}(S) / 2 n(S)$, so $f^{\prime}: S^{\prime} \rightarrow X$ is also extremal. But $f^{\prime}$ can be compressed along the (now embedded) loop $\alpha$, reducing $-\chi^{-}$while keeping $n\left(S^{\prime}\right)$ fixed, thereby contradicting the fact that $f: S \rightarrow X$ was extremal.

Similarly, suppose $\alpha$ is an arc such that $f(\alpha) \cup \beta$ is homotopically trivial in $X$. Let $S^{\prime}$ be a cover of $S$ in which $\alpha$ is embedded. Let $S^{\prime \prime}$ be obtained from $S^{\prime}$ by attaching a 1 -handle $R$ to $\partial \alpha$, and let $f^{\prime \prime}: S^{\prime \prime} \rightarrow X$ be equal to $f^{\prime}$ on $S^{\prime}$, and map the core of $R$ to $\beta$. Then $n\left(S^{\prime \prime}\right)=n\left(S^{\prime}\right)$. However, the union of $\alpha$ with the core of $R$ is an essential embedded loop in $S^{\prime \prime}$ that maps to a homotopically trivial loop in $X$. Hence we can compress this loop, obtaining $f^{\prime \prime \prime}: S^{\prime \prime \prime} \rightarrow X$ with $-\chi^{-}\left(S^{\prime \prime \prime}\right) / 2 n\left(S^{\prime \prime \prime}\right)<-\chi^{-}(S) / 2 n(S)$, thereby contradicting the fact that $f: S \rightarrow X$ was extremal.

A similar argument shows that if a closed surface realizes the Gromov-Thurston norm in its homology class, it is injective. In the sequel, by abuse of notation, we will use the phrase " $S$ is injective" to mean that $f, S$ is incompressible and boundary incompressible. 


\section{Surface subgroups}

\subsection{Graphs of free groups}

Definition 3.1 A graph of groups is a collection of groups indexed by the vertices and edges of a connected graph, together with a family of injective homomorphisms from the edge groups into the vertex groups. Formally, let $\Gamma$ be a connected graph. For each vertex $v$ there is a vertex group $G_{v}$, and for each edge $e$ an edge group $G_{e}$ so that for each inclusion $i: v \rightarrow e$ as an endpoint, there is an injective homomorphism $\varphi_{i}: G_{e} \rightarrow G_{v}$.

The fundamental group $G$ of a graph $\Gamma$ of groups (as above) is defined as follows. Let $G^{\prime}$ be the group generated by all the groups $G_{v}$ and an element $e$ for each (oriented) edge $e$ with relations that each edge element $e$ conjugates the subgroup $i\left(G_{e}\right)$ of $G_{v}$ to the subgroup $j\left(G_{e}\right)$ of $G_{w}$, where $v$ is the initial vertex of $e$ and $w$ is the final vertex, with respect to the choice of orientation on $e$. Let $T$ be a maximal subtree of $\Gamma$. Then define $G$ to be the quotient of $G^{\prime}$ by the normal subgroup generated by elements $e$ corresponding to edges of $T$.

By abuse of notation, we sometimes say that $G$ is a graph of groups with graph $\Gamma$. See eg Serre [14, Section 5.1]. for more details.

In the sequel, let $G$ be a graph of groups with graph $\Gamma$ satisfying the following properties:

(1) Every vertex group $G_{v}$ is free of finite rank

(2) Every edge group $G_{e}$ is cyclic

(3) The graph $\Gamma$ is finite

We say that such a group $G$ is a graph of free groups amalgamated over cyclic subgroups.

\subsection{Hyperbolic groups}

Definition 3.2 A path-metric space $X$ is $\delta$-hyperbolic for some $\delta \geq 0$ if for every geodesic triangle $a b c$, the edge $a b$ is contained in the (metric) $\delta$-neighborhood of the union of edges $a c \cup b c$.

Definition 3.3 A group $G$ with a finite generating set $S$ is word-hyperbolic (or just hyperbolic for short) if the Cayley graph $C_{S}(G)$ is $\delta$-hyperbolic as a path metric space, for some finite $\delta$. 
Hyperbolic groups are introduced in Gromov [9], inspired in part by work of Cannon, Epstein, Rips and Thurston. The theory of hyperbolic groups is vast; the only property of hyperbolic groups we will need is that they do not contain $\mathbb{Z} \oplus \mathbb{Z}$ subgroups or Baumslag-Solitar subgroups. Here the Baumslag-Solitar group $B(p, q)(p, q \neq 0)$ is given by the presentation

$$
B(p, q):=\left\langle a, b \mid b a^{p} b^{-1}=a^{q}\right\rangle
$$

Note that $B(1,1)=\mathbb{Z} \oplus \mathbb{Z}$ as a special case.

\subsection{Construction of surface subgroups}

We are now in a position to state the main theorem of this paper.

Theorem 3.4 Let $G$ be a graph of free groups amalgamated over cyclic subgroups. If $G$ is word-hyperbolic, and $H_{2}(G ; \mathbb{Q})$ is nonzero, then $G$ contains a closed hyperbolic surface subgroup. Furthermore, the unit ball of the Gromov-Thurston norm in $\mathrm{H}_{2}(G ; \mathbb{R})$ is a finite-sided rational polyhedron.

Proof We build a space $X$ with $\pi_{1}(X)=G$ as follows. For each vertex $v$ let $H_{v}$ be a handlebody with $\pi_{1}\left(H_{v}\right)=G_{v}$. For each edge $e$ let $A_{e}$ be an annulus. For each $i: v \rightarrow e$ let $\gamma_{i} \subset X$ be an embedded loop representing the conjugacy class of the generator of $i\left(G_{e}\right)$, and glue the corresponding boundary component of $A_{e}$ to $H_{v}$ along $\gamma_{i}$. The Seifert-van Kampen theorem justifies the equality $\pi_{1}(X) \cong G$. In fact, since each $H_{v}$ and $A_{e}$ is a $K(\pi, 1)$, and since the edge homomorphisms are all injective, the space $X$ itself is a $K(\pi, 1)$. See eg Hatcher [10, Theorem 1B.11, page 92]. Hence $H_{2}(G ; \Lambda)=H_{2}(X ; \Lambda)$ for all coefficient groups $\Lambda$.

Let $E$ denote the union of the cores of the annuli $A_{e}$. Let $V=X-E$ and let $N$ be a regular neighborhood of $E$. The Mayer-Vietoris sequence contains the following exact subsequence

$$
H_{2}(V) \oplus H_{2}(N) \rightarrow H_{2}(X) \rightarrow H_{1}(V \cap N) \rightarrow H_{1}(V) \oplus H_{1}(N)
$$

Since $H_{2}(V)=H_{2}(N)=0$, it follows that an element of $H_{2}(X)$ is determined by its image in $H_{1}(V \cap N)$.

Let $A$ be a nonzero class in $H_{2}(X)$ represented by a map of a closed surface $f: S \rightarrow X$. If we make $f$ transverse to the core of each $A_{e}$ and adjust by a homotopy, we can assume that $S_{e}:=f^{-1}\left(A_{e}\right)$ is a union of subsurfaces of $S$ each mapping properly to $A_{e}$. If $S_{e}^{i}$ is a component of $S_{e}$, the degree of $f: S_{e}^{i} \rightarrow A_{e}$ is equal to the number of times $\partial S_{e}^{i}$ winds (with multiplicity) around either boundary component of $A_{e}$. If 
some $S_{e}^{i}$ maps to some $A_{e}$ with degree 0 , compress a suitable subsurface of $S_{e}^{i}$ and push it off $A_{e}$ by a homotopy.

For each $v$, let $\gamma_{1}, \cdots, \gamma_{m}$ denote the set of loops in $H_{v}$ that are the boundaries of components of the various $A_{e}$. The surface $S_{v}:=f^{-1}\left(H_{v}\right)$ maps to $H_{v}$ with boundary wrapping various times around the various $\gamma_{i}$. Let $n_{i} \in \mathbb{Z}$ be such that $S_{v}$ is an admissible surface bounding $\sum n_{i} \gamma_{i}$. Note that the $n_{i}$ (for various $v$ ) are determined by the homology class $A$, and are precisely the coefficients of the element $\partial A \in H_{1}(V \cap N)$ with respect to a basis for $H_{1}(V \cap N)$ consisting of the various $\gamma_{i}$.

For each $v$, let $g_{v}: T_{v} \rightarrow X$ be an extremal surface for $\sum n_{i} g_{i}$. Note that $\partial T_{v}$ represents $n_{v} \sum n_{i} g_{i}$ in $H_{1}(V \cap N)$ for some integer $n_{v}$. If the various $T_{v}$ could be glued together along their boundary components compatibly with $\Gamma$, the components of the resulting surface would be injective, and their union would map to $X$, representing a multiple of the class $A$ in $H_{2}(X)$. If $G$ is word-hyperbolic, we will show how to construct suitable covers of the $T_{v}$ that can in fact be glued up. Finite covers of extremal surfaces are also extremal, so ultimately this will let us construct a surface mapping to $X$ and representing a multiple of the class $A$, that is built by gluing extremal surfaces as above.

Lemma 3.5 Let $S$ be an orientable surface with nonempty boundary components $\partial_{i} S$. For $N \in \mathbb{Z}$, let $\phi: \partial_{i} \rightarrow \mathbb{Z} / N \mathbb{Z}$ be some function. If $\sum_{i} \phi\left(\partial_{i}\right)=0 \in \mathbb{Z} / N \mathbb{Z}$ then $\phi$ extends to a function $\pi_{1}(S) \rightarrow \mathbb{Z} / N \mathbb{Z}$ whose kernel defines a regular cover $S^{\prime}$ of $S$ with the property that each boundary component $\partial_{i j}$ in the preimage of $\partial_{i}$ maps to $\partial_{i}$ with degree equal to the order of $\phi\left(\partial_{i}\right)$ in $\mathbb{Z} / N \mathbb{Z}$.

Proof Homomorphisms from $\pi_{1}(S)$ to abelian groups are exactly those that factor through the abelianization $H_{1}(S)$. The components $\partial_{i}$ determine elements of $H_{1}(S)$ that are subject only to the relation $\sum \partial_{i}=0 \in H_{1}(S)$. This follows directly from the exact sequence in relative homology

$$
H_{2}(S) \rightarrow H_{2}(S, \partial S) \rightarrow H_{1}(\partial S) \rightarrow H_{1}(S)
$$

together with the fact that $H_{2}(S)=0$ and $H_{2}(S, \partial S)=\mathbb{Z}$.

The other statements are standard facts from the theory of covering spaces.

By invoking Lemma 3.5 repeatedly, we will construct covers of the $T_{v}$ that can be glued up over the various $A_{e}$ one by one. Let $e$ be an edge, and $v, w$ the end vertices. Let $\gamma \in H_{v}$ and $\delta \in H_{w}$ be the loops along which the boundary components of $A_{e}$ are attached. Suppose we have surfaces $T=T_{v}$ and $U=U_{w}$ for some $v, w$ mapping to 
$X$ and subsets $\partial_{\gamma} T, \partial_{\delta} U$ of the boundary components that map to $\gamma$ and $\delta$ respectively. By Lemma 2.6 we can assume that each component of $\partial_{\gamma} T$ maps to $\gamma$ with positive degree, and similarly for $\partial_{\delta} U$. Note that we should allow the possibility that $T=U$.

Assume for the moment that $\chi(T)<0$ and $\chi(U)<0$. If $Z$ is any connected surface of negative Euler characteristic, then $Z$ admits a finite cover with positive genus. Furthermore, if $Z$ has positive genus, then $Z$ admits a degree 2 cover $Z^{\prime}$ such that every boundary component of $Z$ has exactly two preimages in $Z^{\prime}$, each of which maps with degree 1. Applying this observation to $T$ and $U$, after replacing $T$ and $U$ by finite covers if necessary, we can assume that the components of $\partial_{\gamma} T$ come in pairs that each map to $\gamma$ with the same degree, and similarly for the components of $\partial_{\delta} U$.

Let $N$ be the least common multiple of the degrees of maps from components of either $\partial_{\gamma} T$ or $\partial_{\delta} U$ to $\gamma$ or $\delta$. We will define homomorphisms $\phi: \pi_{1}(T) \rightarrow \mathbb{Z} / N \mathbb{Z}$ and $\psi: \pi_{1}(U) \rightarrow \mathbb{Z} / N \mathbb{Z}$ as follows. If $\nu, v^{\prime}$ are a pair of components of $\partial_{\gamma} T$ mapping to $\gamma$ with the same degree $d$, then define $\phi(v)=d$ and $\phi\left(v^{\prime}\right)=-d$, and define $\psi$ similarly on pairs of components of $\partial_{\delta} U$. Note that $\phi, \psi$ may be extended to have the value 0 on components of $\partial T$ and $\partial U$ not appearing in $\partial_{\gamma} T$ or $\partial_{\delta} U$. Let $T^{\prime}, U^{\prime}$ be the corresponding covers. Then by construction, every component of $\partial_{\gamma} T^{\prime}$ maps to $\gamma$ with degree $N$, and every component of $\partial_{\delta} U^{\prime}$ maps to $\delta$ with degree $N$, so $U^{\prime}$ and $T^{\prime}$ can be glued up along $\partial_{\gamma} T^{\prime}$ and $\partial_{\delta} U^{\prime}$ and their maps to $X$ extended over $A_{e}$. Proceeding inductively, we can construct a surface $S^{\prime}$ and a map $f^{\prime}: S^{\prime} \rightarrow X$ representing some integral multiple of the class $A$. Observe that $S^{\prime}$ is made by gluing extremal surfaces, which are incompressible and boundary incompressible by Lemma 2.7. From the Seifert van-Kampen theorem it follows that every component of $S^{\prime}$ is injective.

If $\chi(T)=0$ for some (possibly intermediate) surface $T$, then $T$ consists of a union of annuli. If not every component $T$ is being glued up to $U$, one can still take covers of these annuli as above and glue up. The only potentially troublesome case is when $T=U$, and the free boundary components of $T$ are being glued up to each other. But in this case, $G$ contains a Baumslag-Solitar group, and is therefore not hyperbolic. This proves the first part of the theorem.

We now prove the statement about Gromov-Thurston norms. First, observe that any surface $Z$ obtained by gluing extremal surfaces realizes the Gromov-Thurston norm in its homology class. For, let $Z^{\prime}$ be another surface with $[Z]=\left[Z^{\prime}\right]$. As in the first part of the proof, we can compress any simple loop in $Z^{\prime}$ that maps with degree 0 to some $A_{e}$. Then by cutting both $Z$ and $Z^{\prime}$ along the $A_{e}$ we decompose them into subsurfaces $Z_{v}, Z_{v}^{\prime}$ that represent the same relative homology class in each $H_{v}$. By 
the definition of extremal, $-\chi^{-}\left(Z_{v}\right) \leq-\chi^{-}\left(Z_{v}^{\prime}\right)$ for each $v$, so $-\chi^{-}(Z) \leq-\chi^{-}\left(Z^{\prime}\right)$ as claimed. Moreover we have shown above that every rational homology class is projectively represented by a surface obtained by gluing extremal surfaces. Hence the Gromov-Thurston norm of a rational homology class $A$ in $G$ is equal to the sum of the scl norms of the components of $\partial A$ in the various $G_{v}$, up to a normalizing factor of 4 .

For each $H_{v}$, let $B_{v}^{\prime}$ be the subspace of $Z_{1}\left(G_{v}\right)$ (the space of group 1-cycles) spanned by the $\gamma_{i}$ along which various $A_{e}$ are attached, and let $B_{v}$ be the kernel of the natural map $B_{v}^{\prime} \rightarrow H_{1}\left(G_{v}\right)$. The boundary map in the Mayer-Vietoris sequence defines an integral linear injection $H_{2}(X) \stackrel{\partial}{\rightarrow} \bigoplus_{v} B_{v}$ with components $\partial_{v}(A) \in B_{v}$, and by the discussion above,

$$
\|A\|_{1}=4 \cdot \sum_{v} \operatorname{scl}\left(\partial_{v} A\right)
$$

Since each $\partial_{v}$ is an integral linear map, and the scl pseudo-norm on each $B_{v}$ is a rational piecewise-linear function, the $L^{1}$ norm (ie the Gromov-Thurston norm) on $H_{2}(X ; \mathbb{R})$ is a piecewise rational linear function. Since $G$ is hyperbolic, the unit ball is a (nondegenerate) finite sided rational polyhedron.

Remark 3.6 If $G$ is not necessarily word-hyperbolic, it is nevertheless true (essentially by the argument above) that the Gromov-Thurston pseudo-norm on $H_{2}(G ; \mathbb{R})$ is piecewise rational linear. Moreover, the same argument shows that for any $G$ obtained as a graph of free groups amalgamated over cyclic subgroups, and for any homology class $A \in H_{2}(G ; \mathbb{Q})$, either some multiple of $A$ is represented by an injective closed hyperbolic surface, or $\|A\|_{1}=0$.

Remark 3.7 In fact, if $A$ is a nontrivial homology class in $G$ with $\|A\|_{1}=0$, the nonabelian Baumslag-Solitar case does not arise. For, $H_{2}(B(n, m) ; \mathbb{Z})=0$ whenever $n \neq m$, and therefore no nontrivial rational homology class in $G$ can be represented by a map of a surface that factors through a Baumslag-Solitar group. Hence every class $A$ in $G$ as above with $\|A\|_{1}=0$ is projectively realized by a $\mathbb{Z} \oplus \mathbb{Z}$ subgroup.

Remark 3.8 If $M$ is a compact 3-manifold, every integral class $A$ in $H_{2}\left(\pi_{1}(M)\right.$; $\left.\mathbb{Z}\right)$ is represented by an embedded surface $S$ that realizes the infimum of $-\chi^{-}$in its projective class. It follows that the Gromov-Thurston norm (with Gromov's normalization) takes on values in $4 \mathbb{Z}$ on $H_{2}\left(\pi_{1}(M) ; \mathbb{Z}\right)$. This by itself ensures that the unit ball is a rational polyhedron. However, for $G$ a graph of free groups as above, the GromovThurston norm can take on rational values with arbitrary denominators on elements of $H_{2}(G ; \mathbb{Z})$, so the polyhedrality of the norm is more subtle. See [3, Section 4.1.9]. 
Remark 3.9 Since this work was circulated, various people, including Jason Manning, Eduardo Martinez-Pedroza, Henry Wilton and Cameron Gordon, have extended the main result, using it to construct surface subgroups in certain kinds of limit groups. I am grateful to Jason Manning for pointing this out.

Acknowledgments While writing this paper I was partially supported by NSF grant DMS 0707130. I would like to thank Mladen Bestvina, Benson Farb, Lars Louder and Dongping Zhuang for comments and some discussion. I am also very grateful to the anonymous referee for some quick and helpful corrections. The work in this paper builds largely on work done in [4] which benefited greatly from many discussions with Jason Manning.

\section{References}

[1] C Bavard, Longueur stable des commutateurs, Enseign. Math. (2) 37 (1991) 109-150 MR1115747

[2] M Bestvina, Questions in geometric group theory, available at http://www. math. utah. edu/ bestvina

[3] D Calegari, $s c l$, monograph, available at http://www.its.caltech.edu/ dannyc

[4] D Calegari, Stable commutator length is rational in free groups arXiv:0802.1352

[5] D Gabai, Foliations and the topology of 3-manifolds, J. Differential Geom. 18 (1983) 445-503 MR723813

[6] S M Gersten, Cohomological lower bounds for isoperimetric functions on groups, Topology 37 (1998) 1031-1072 MR1650363

[7] C M Gordon, D D Long, A W Reid, Surface subgroups of Coxeter and Artin groups, J. Pure Appl. Algebra 189 (2004) 135-148 MR2038569

[8] M Gromov, Volume and bounded cohomology, Inst. Hautes Études Sci. Publ. Math. (1982) 5-99 MR686042

[9] M Gromov, Hyperbolic groups, from: "Essays in group theory", Math.. Sci. Res. Inst. Publ. 8, Springer, New York (1987) 75-263 MR919829

[10] A Hatcher, Algebraic topology, Cambridge University Press (2002) MR1867354

[11] J Hempel, 3-Manifolds, Ann. of Math. Studies 86, Princeton University Press (1976) MR0415619

[12] S Mac Lane, Homology, Die Grund. der math. Wissenschaften 114, Academic Press Publishers, New York (1963) MR0156879

[13] P Scott, Subgroups of surface groups are almost geometric, J. London Math. Soc. (2) 17 (1978) 555-565 MR0494062 
[14] J-P Serre, Trees, Springer Monographs in Math., Springer, Berlin (2003) MR1954121 Translated from the French original by J Stillwell, Corrected 2nd printing of the 1980 English translation

[15] W P Thurston, A norm for the homology of 3-manifolds, Mem. Amer. Math. Soc. 59 (1986) i-vi and 99-130 MR823443

Department of Mathematics, Caltech

Pasadena CA, 91125

dannyc@its.caltech.edu

Proposed: Benson Farb

Received: 4 April 2008

Seconded: Joan Birman and Dave Gabai

Revised: 9 June 2008

Geometry $\&$ Topology, Volume 12 (2008) 